\title{
Anti-Wrinkle Efficacy of Cross-Linked Hyaluronic Acid-Based Microneedle Patch with Acetyl Hexapeptide-8 and Epidermal Growth Factor on Korean Skin
}

\author{
Ji Hae An, Hee Jung Lee, Moon Soo Yoon, Dong Hyun Kim \\ Department of Dermatology, CHA Bundang Medical Center, CHA University School of Medicine, Seongnam, Korea
}

Background: Hyaluronic acid (HA)-based microneedle patch has recently been studied for wrinkle improvement. Cross-linked HA (CLHA) is widely used in dermal fillers. Acetyl hexapeptide-8 (AHP-8) and epidermal growth factor (EGF) are used for cosmetic ingredients. Objective: This study aimed to verify the efficacy of the CLHA/HA-based patch with microstructure (microneedle patch) containing AHP-8 or EGF. Methods: A total of 52 Korean females were enrolled in a double-blind, randomized, controlled, splitface trial. The subjects were divided into 3 groups: (1) microneedle patch alone, (2) microneedle patch/AHP-8, and (3) microneedle patch/EGF. The treatment was applied on the periorbital and nasolabial fold area for 4 hours to completely dissolve the microstructures once per week for 29 days. Evaluations, including photodamage scoring, image analysis with Antera 3D ${ }^{\circledR}$ (Miravex, Ireland), skin hydration measurement, and adverse effect assessments, were performed at each visit. Results: Fifty subjects (96.2\%) completed this clinical study. On day 29 after application, statistically significant improvements in wrinkle and skin hydration were observed in all groups $(p<0.01)$. Treatment with microneedle patch/AHP-8 and microneedle patch/EGF showed statisti-

Received August 23, 2018, Revised October 18, 2018, Accepted for publication November 2, 2018

Corresponding author: Dong Hyun Kim, Department of Dermatology, CHA Bundang Medical Center, CHA University, 59 Yatap-ro, Bundang-gu, Seongnam 13496, Korea. Tel: 82-31-780-5243, Fax: 82-31-780-5247, E-mail: terios92@hanmail.net

ORCID: https://orcid.org/0000-0003-3394-2400

This is an Open Access article distributed under the terms of the Creative Commons Attribution Non-Commercial License (http://creativecommons. org/licenses/by-nc/4.0) which permits unrestricted non-commercial use, distribution, and reproduction in any medium, provided the original work is properly cited.

Copyright $($ c The Korean Dermatological Association and The Korean Society for Investigative Dermatology cally significant improvements in wrinkle compared with microneedle patch alone $(p<0.05)$. No serious adverse effects were noted. Conclusion: Combination of CLHA-based microneedle patch and functional cosmetic ingredients can improve wrinkle with minimal discomfort. (Ann Dermatol 31(3) $263 \sim 271,2019$ )

-Keywords-

Acetyl hexapeptide-8, Cross-linked hyaluronic acid, Epidermal growth factor, Hyaluronic acid, Microneedle patch

\section{INTRODUCTION}

Skin aging is associated with dryness and decrease of skin elasticity and flexibility. Hyaluronic acid (HA) is an endogenous linear polysaccharide that consists of repeating $\mathrm{N}$-acetyl-D-glucosamine and D-glucuronic acid units. Approximately $50 \%$ of the total body $\mathrm{HA}$ is normally found in the extracellular matrix of the dermis of the skin; HA organizes and maintains its structure to reduce the appearance of wrinkles. This is due to the high water-binding capacity and swelling properties of $\mathrm{HA}^{1}$. Moreover, the safe, biocompatible, and biodegradable properties of HA make it applicable to safe cosmetic products and in the field of dermatology $\mathrm{y}^{2,3}$.

However, HA possesses poor mechanical properties that are easily degraded by hyaluronidase and free radicals. To improve the sustainability and mechanical limits, $\mathrm{HA}$ is covalently and chemically cross-linked to form a three-dimensional cross-linked HA (CLHA) to prolong the duration of its stay in the skin compared with non-CLHA ${ }^{4,5}$. This is the reason that CLHA hydrogel is widely employed for injectable dermal filler. However, dermal filler inject- 
ion entails pain and is highly likely to cause adverse effects. Moreover, topical cosmetic products with HA have low absorption rates. The HA- and CLHA-based microneedle patch, which has a higher delivery rate than do topical cosmetic products, is composed of the same functional ingredients as dermal fillers. It can be easily attached and is less likely to cause pain and adverse effects compared with dermal fillers. Therefore, microneedle patch has received considerable attention ${ }^{6}$. Choi et al. ${ }^{7}$ evaluated the ability of microneedle patch containing $\mathrm{HA}$ to improve wrinkles around the eyes. In another study, Kim et al. ${ }^{8}$ added retinoic acid or ascorbic acid to microneedle patch and evaluated their anti-wrinkle effects. However, most microneedle patches use non-CLHA ${ }^{7-10}$. To the best of our knowledge, CLHA-based microneedle patch has not yet been investigated.

In this study, we conducted a randomized, double-blind, controlled, split-face, clinical examination of the patch with micro structure (microneedle patch), which was fabricated with mixed CLHA and $\mathrm{HA}$. We aimed to verify the combination efficacy of CLHA and functional cosmetic ingredients by adding acetyl hexapeptide-8 (AHP-8) and epidermal growth factor (EGF) to the microneedle patch.

\section{MATERIALS AND METHODS}

\section{Microneedle patch fabrication}

The microneedle patch was developed and produced by Endoderma Co., Ltd. (Seongnam, Korea). The patch consists of a microstructure film and a hydrocolloid pad. The film was fabricated through a micro-molding technology, and the solution was prepared by mixing CLHA and HA. AHP-8 (Lipotec SAU, Barcelona, Spain) at a concentration of $0.005 \%$ (w/w) and EGF (CHA Meditech Co., Ltd., Daejeon, Korea) at a concentration of $0.005 \%(w / w)$ have also been combined with this soloution. The microstructures of the microneedle patch were analyzed using an optical microscope (Eclipse 80i; Nikon, Tokyo, Japan).

\section{Penetration and dissolution test of patch}

The microneedle patch was pushed with a force of $30 \mathrm{~N}$ for 10 seconds by a compression force station (HAP-0015; Hanatech, Seoul, Korea) into a 5-mm thickness of porcine skin (Cronex, Seoul, Korea). Each penetrated site was dyed with $0.4 \%$ Trypan Blue (Sigma-Aldrich, Darmstadt, Germany) and observed through an optical microscope (Sv-35; Sometech, Seoul, Korea). Detached microneedle patches were analyzed using a scanning electron microscope (SEM, JSM-7001F; JEOL, Tokyo, Japan) after 10 seconds, 15 minutes, and 30 minutes of application.

\section{Subjects}

All procedures performed in studies involving human subjects were in accordance with the ethical standards of the Institutional Review Board (IRB) of CHA Bundang Medical Center (IRB file no. 2016-12-038) and with the 1964 Helsinki declaration and its later amendments or comparable ethical standards. Informed consent was obtained from all individual subjects included in the study. In addition, we received the patient's consent form about publishing all photographic materials. Healthy female volunteers with a photodamage score $^{11,12}$ ranging between 2 and 6 were enrolled. Those who had skin diseases and other disorders, were pregnant or breastfeeding, and had participated in the same or similar clinical trial within the last three months were excluded.

\section{Study method}

All microneedle patches used in this study contained the same mixture of CLHA and HA. The microneedle patch with AHP-8 and that with EGF were used to verify the combination efficacy of functional cosmetic ingredients. All test products were identically packaged so that the researchers and subjects could not discriminate which functional ingredients the products contained. The subjects were randomly divided into (1) microneedle patch alone, (2) microneedle patch/AHP-8, and (3) microneedle patch/ EGF groups by the randomization table. Also, subjects were trained on how to apply the products by the same researcher to make the same amount of product delivers as far as possible.

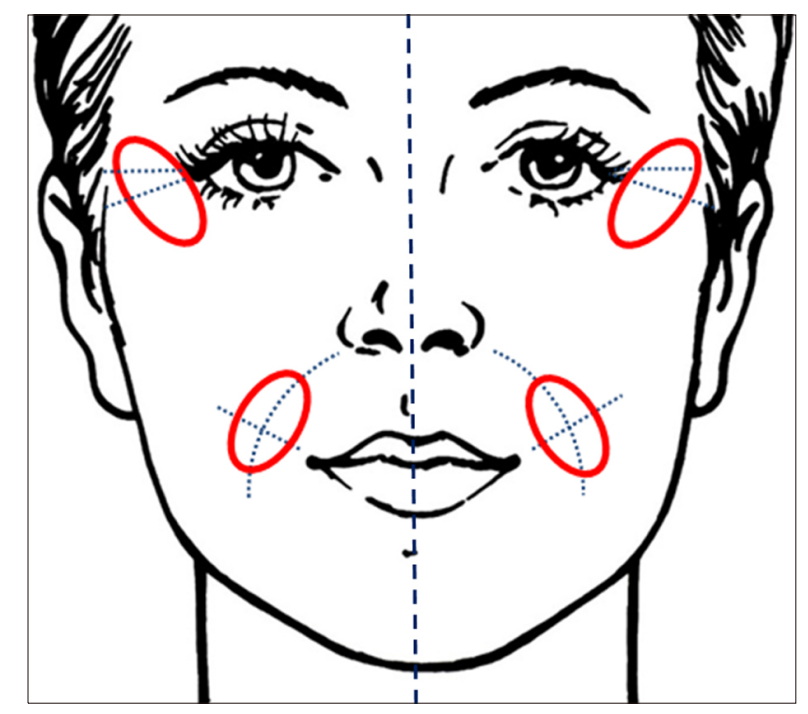

Fig. 1. The image of split-face experiment. Red circle: patch attachment site. 
Each trained subjects applied each test products on the left and right sides of their periorbital and nasolabial fold areas once per week for 29 days for the split-face test (Fig. 1). The applied site was designated as the area $1 \mathrm{~cm}$ lateral to lateral canthus and the center of virtual line connecting nasal ala and oral commissure. When applying the microneedle patch into the skin, it was attached for 4 hours to fully dissolve the microstructures. The Antera 3D ${ }^{\circledR}$ (Miravex, Dublin, Ireland) was used to evaluate the anti-wrinkle effect $^{13}$, and the Corneometer ${ }^{\mathbb{R}}$ (Corneometer CM 825; Courage + Khazaka, Cologne, Germany) was used to evaluate the improvement in skin moisture content. In addition, three dermatologists met as a panel, discussed each of the photographs of the subjects, and assigned each subject a wrinkle grade between 0 and 7 according to wrinkle severity. Eight photographic standards illustrate increasing severity of wrinkles, where 0 represents none; 1 , none/ mild; 2 , mild; 3, mild/moderate; 4, moderate; 5, moderate/ severe; 6 , severe; and 7 , very severe ${ }^{11,12}$. The assessment of safety was performed on the degree of adverse effects including erythema, edema, itching, stinging, tightness. On day $0,1,3,5,8$, and 29 of the application, the efficacy and safety were assessed at the same site with the same method as the first visit.

\section{Statistical analysis}

Statistical analyses were performed using IBM SPSS Statistics ver. 23.0 (IBM Co., Armonk, NY, USA). The evaluation variables of the efficacy before and after application were analyzed with the Wilcoxon signed-rank test, and the difference between groups was compared using the Mann-Whitney test to evaluate statistical differences. We considered $p$-values $<0.05$ to be statically significant.

\section{RESULTS}

\section{Fabricated microstructures and characterization of the inserted and dissolved microneedle patch}

The size of the microneedle patch was $1.5 \times 2.0 \mathrm{~cm}^{2}$. The observed cone and cylinder microstructure were shaped with approximately $600-\mu \mathrm{m}$ intervals, $260-\mu \mathrm{m}$ height, and $100-\mu \mathrm{m}$ diameter. The uniformly arrayed microstructures were successfully fabricated on the patch (Fig. 2). Moreover, the structures were made to form hexagonal patterns having equal distances between adjacent microstructures to achieve uniform penetration forces in geometries, when they are pressed into the skin $^{14}$.

The blue dots on the surface of the pierced porcine skin
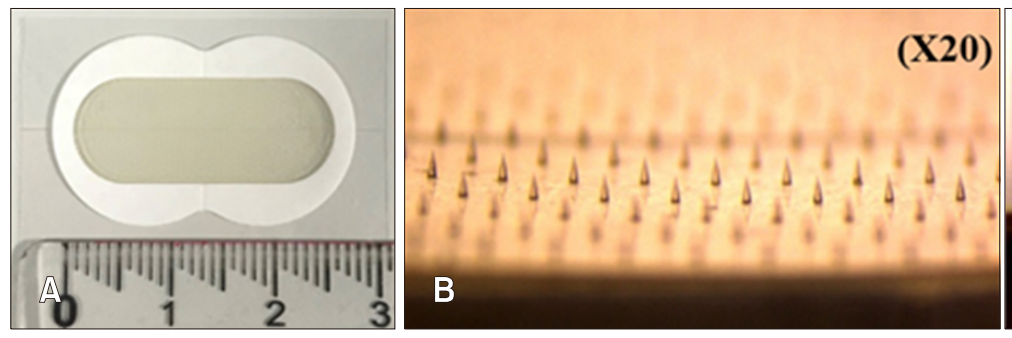

X20)

(X50)

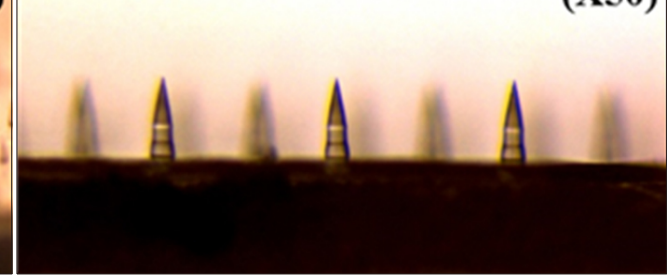

Fig. 2. (A) Microneedle patch image, (B) microscopic images of microstructures $(\times 20, \times 50)$.
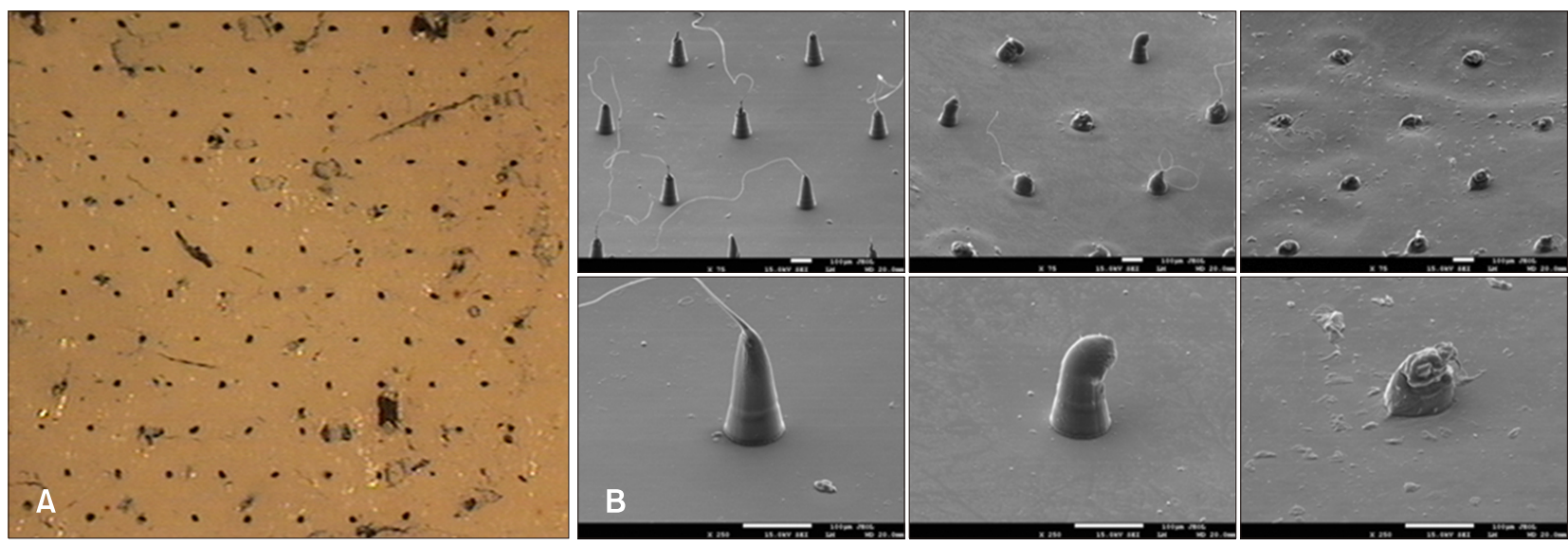

Fig. 3. (A) Penetrated and dyed microscopic image of porcine skin. (B) Scanning electron microscope images of dissolved microstructures depending on the application time differences (from the left side) 10 seconds, 15 minutes, and 30 minutes, respectively. 
after microneedle patch application represented the penetration sites of the microstructures. The microstructure showed successful penetration by $99 \% \pm 1 \%$ (Fig. 3A), which means that the structure had sufficient solidity to endure the pressing force when it is pushed into the skin. Furthermore, the dissolving ratio of the inserted microstructures was demonstrated after the patch application. The microstructures on the detached microneedle patch were differently dissolved and partially broken depending on the patch application time (Fig. 3B). After 2 hours of contact, $80.39 \%$ of the microstructures were dissolved, indicating that the microstructure needs enough time to be released by water absorption of $\mathrm{HA}$. This is because HA can bind to water as a viscous substance that can assist in turgor and hydration of the skin to reduce wrinkles and dehydration ${ }^{15-17}$. Therefore, the application time of this product has been recommended to be more than 4 hours.

\section{Clinical characteristics of the subjects}

A total of 52 healthy Korean females were enrolled as volunteers. Two subjects later decided to voluntarily withdrew from the study. The subjects were divided into 3
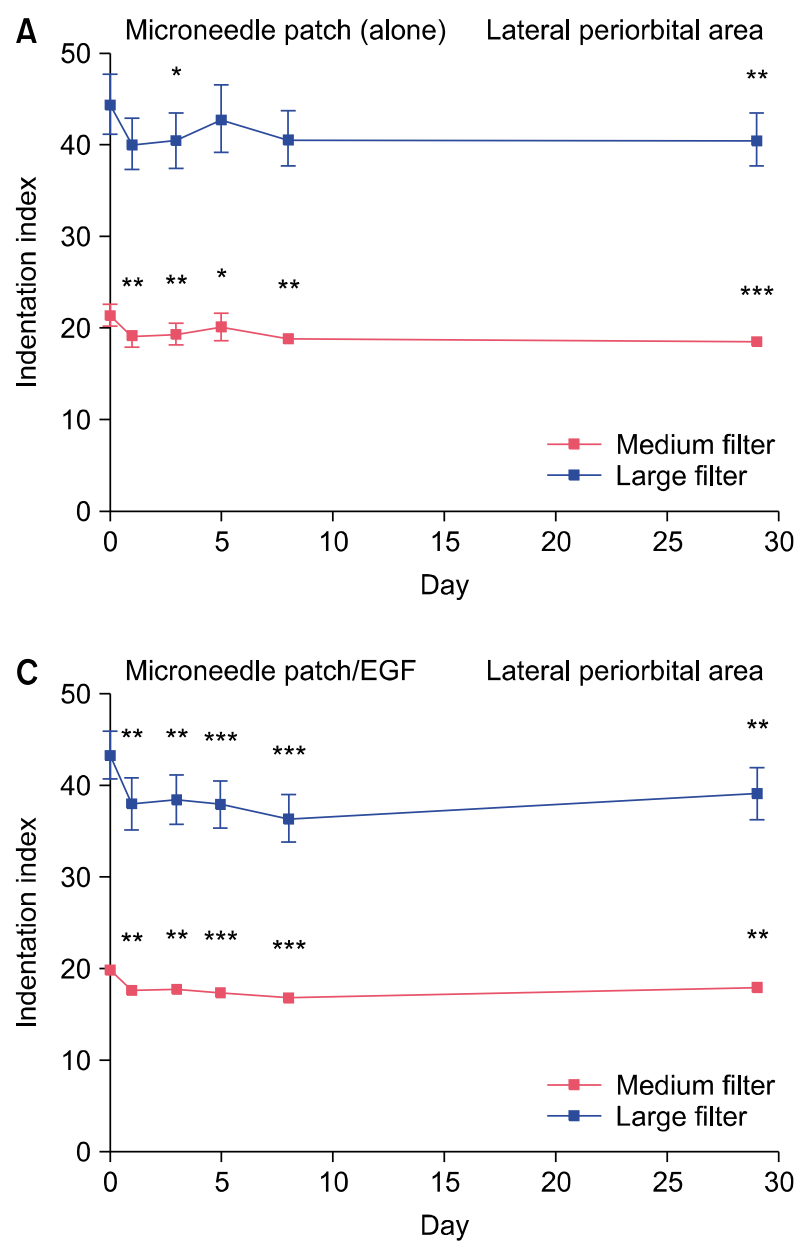

groups: (1) microneedle patch alone, (2) microneedle patch/ AHP-8, and (3) microneedle patch/EGF. The mean age of these subjects was $45.02 \pm 4.85$ years, and their age ranged from 37 to 57 years.

\section{Assessment of the anti-wrinkle effects}

In a wrinkle assessment using the Antera $3 \mathrm{D}^{\circledR}$, two filters were used for measurement. A medium filter is appropriate to analyze fine to moderate wrinkles from $0.5 \mathrm{~mm}$ to $2 \mathrm{~mm}$ in size, and a large filter is appropriate to analyze deep wrinkles less than $3 \mathrm{~mm}$ in size ${ }^{18}$. Analysis of fine to moderate wrinkles around the eyes showed significant improvements in the appearance of wrinkles starting at day 1 after the application compared with before the application for all three products. The degree of improvement was $12.5 \%$ to $14.6 \%$ for microneedle patch/AHP-8, $10.2 \%$ to $15.3 \%$ for microneedle patch/EGF, and $5.9 \%$ to $12.0 \%$ for microneedle patch alone. Analysis of deep wrinkles around the eyes showed significant improvements starting at day 1 after the application of microneedle patch/AHP-8, and microneedle patch/EGF, and the degree of improvement was $12.3 \%$ to $15.2 \%$ and $11.0 \%$ to $15.9 \%$, respectively

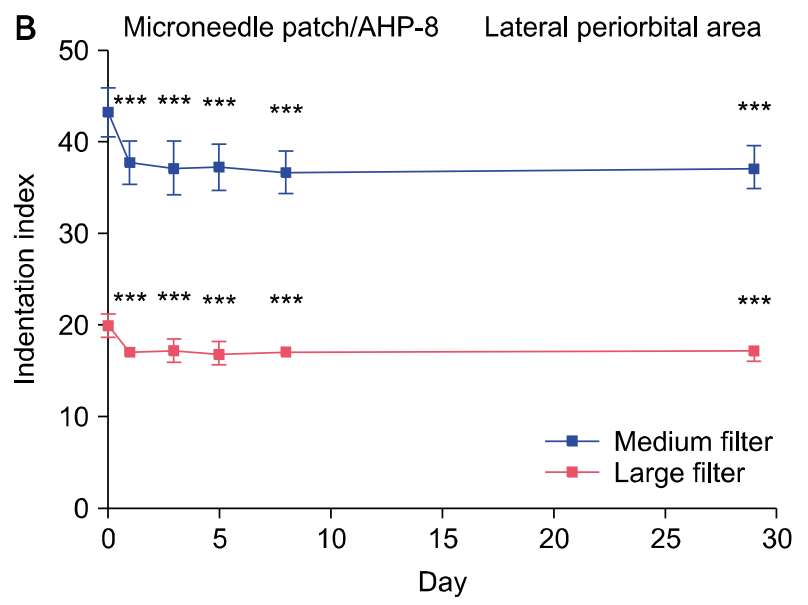

Fig. 4. Mean change in indentation index (medium and large filter) of the Antera $3 D^{\mathbb{R}}$ using (A) microneedle patch alone, (B) microneedle patch/acetyl hexapeptide-8 (AHP-8), and (C) microneedle patch/epidermal growth factor (EGF) on the lateral periorbital area. ${ }^{*} p<0.05,{ }^{* *} p<0.01,{ }^{* * *} p<0.001$, statistically significant compared with baseline. 
(Fig. 4).

Analysis of fine to moderate wrinkles around the nasolabial area showed significant improvements starting at day 1 after the application for all three products. The degree of improvement was $7.6 \%$ to $13.6 \%$ for microneedle patch/ AHP-8, $8.1 \%$ to $13.9 \%$ for microneedle patch/EGF, and $11.7 \%$ to $14.4 \%$ for microneedle patch alone. Analysis of deep wrinkles around the nasolabial area also showed significant improvements starting at day 1 after the application. The degree of improvement was $8.2 \%$ to $15.9 \%$ for microneedle patch/AHP-8, $10.4 \%$ to $16.3 \%$ for microneedle patch/EGF, and $9.9 \%$ to $15.1 \%$ for microneedle patch alone (Fig. 5).

For comparison between products, fine to moderate and deep wrinkles around the periorbital area were analyzed. Analysis of fine to moderate wrinkles around the periorbital area showed that microneedle patch/AHP-8 had significantly higher improvements than microneedle patch alone $(14.6 \%$ and $5.9 \%$, respectively) ( $p<0.05$, Fig. $6 \mathrm{~A})$. Analysis of deep wrinkles around the same area showed that microneedle patch/AHP-8 and microneedle patch/ EGF had significantly higher improvements $(13.6 \%$ and
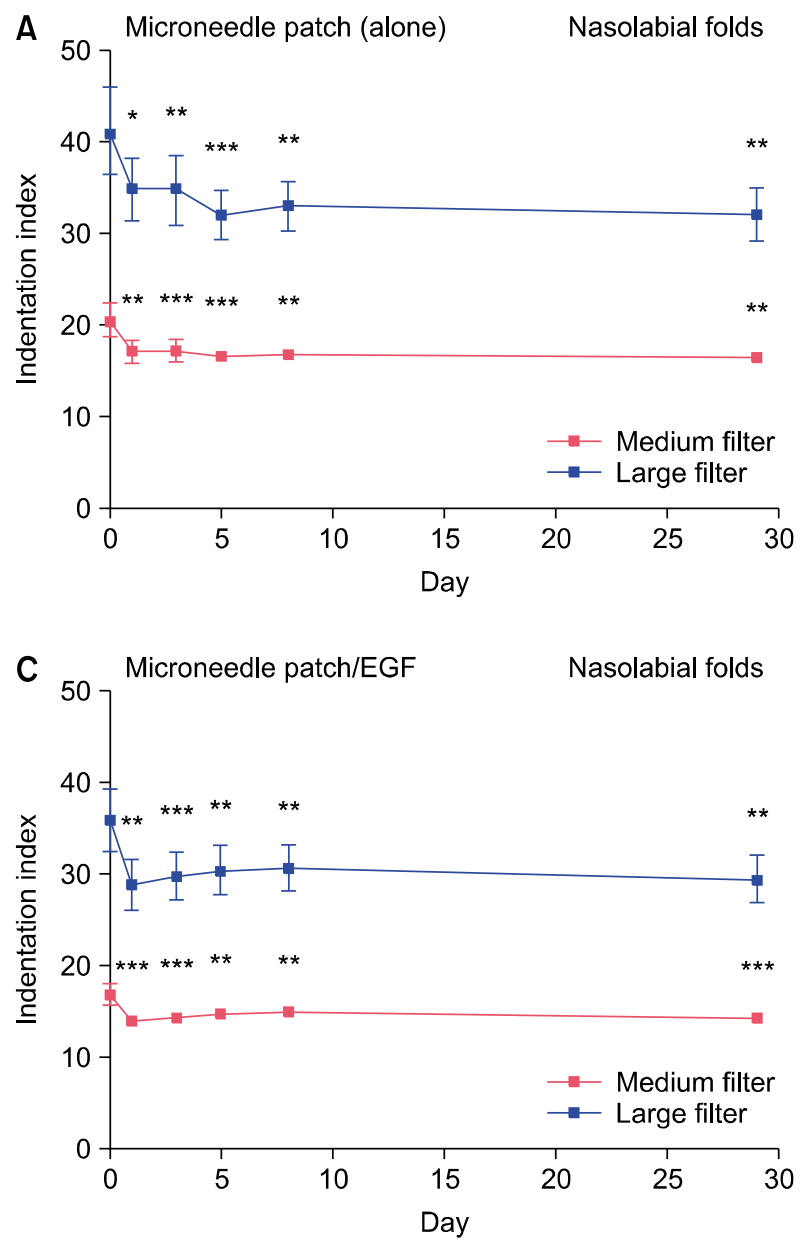

$12.9 \%$, respectively) than microneedle patch alone $(3.5 \%)$ at day 5 after the application ( $p<0.05$, Fig. 6B). No significant difference was found in the degree of improvement between the products in nasolabial fold.

\section{Changes in photodamage score}

The photodamage scores were $3.32 \pm 0.12$ at baseline and $3.08 \pm 0.13$ at day 29 after the application of microneedle patch/AHP-8, and a $7.3 \%$ improvement $(p<0.01)$ was noted. The photodamage score decreased by $7.4 \%$ from $3.32 \pm 0.12$ to $3.07 \pm 0.1$ at day 29 after the application of microneedle patch/EGF $(p<0.01)$. The photodamage score decreased by $5.8 \%$ from $3.48 \pm 0.12$ to $3.28 \pm 0.13$ at day 29 after the application of microneedle patch alone $(p<0.01$, Fig. 7). No significant difference was found in the degree of improvement between the products.

\section{Improvement in skin moisture content}

Skin hydration values were $61.0 \pm 3.3$ at baseline and $68.9 \pm 2.7$ at day 29 after the application of microneedle patch/AHP-8, and a $13.0 \%$ improvement $(p<0.01)$ was noted. And skin hydration value increased by $12.9 \%$ from

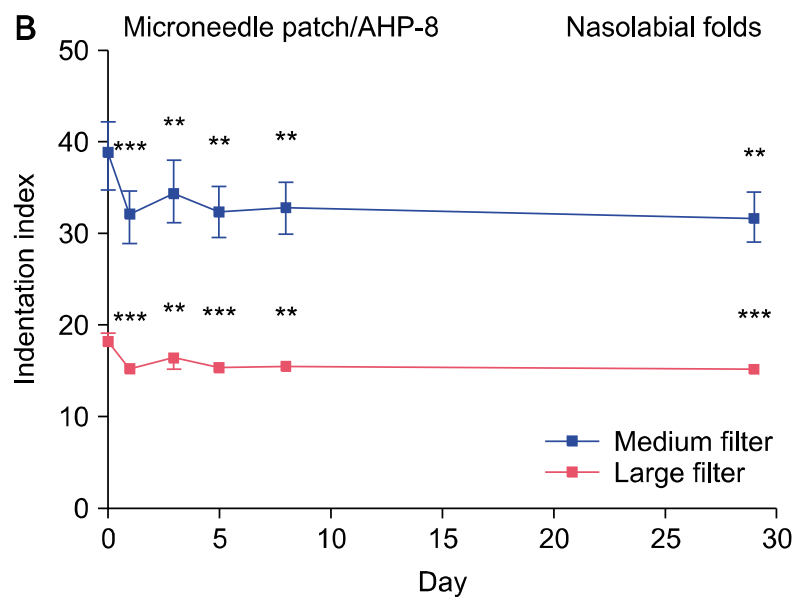

Fig. 5. Mean change in indentation index (medium and large filter) of the Antera $3 \mathrm{D}^{\circledR}$ using (A) microneedle patch alone, (B) microneedle patch/acetyl hexapeptide-8 (AHP-8), and (C) microneedle patch/epidermal growth factor (EGF) on the nasolabial folds. ${ }^{*} p<0.05,{ }^{* *} p<0.01,{ }^{* * *} p<0.001$, statistically significant compared with baseline. 

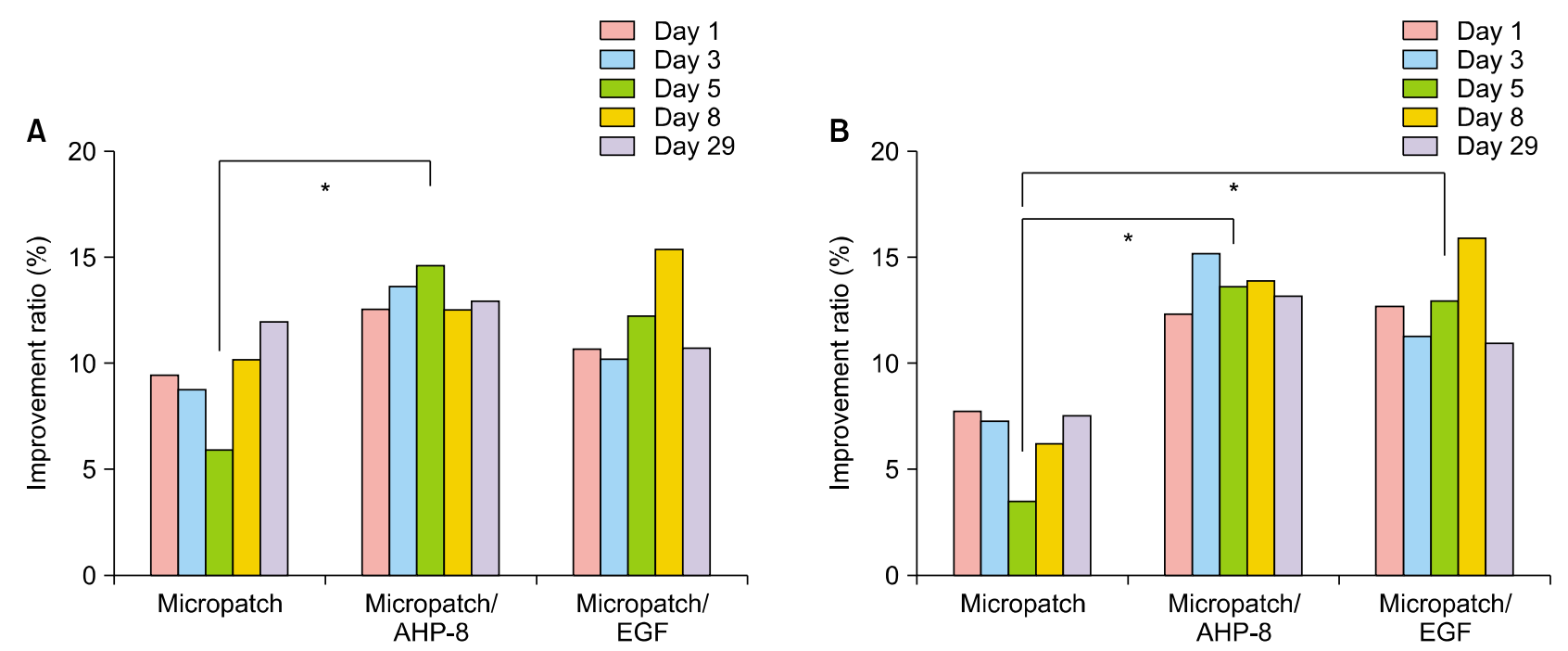

Fig. 6. Improvement ratio of indentation index (medium $[\mathrm{A}]$ and large $[\mathrm{B}]$ ) of the Antera $3 \mathrm{D}^{\circledR}$ on the lateral periorbital area at day 29 after the first application of microneedle patch, microneedle patch/acetyl hexapeptide-8 (AHP-8), and microneedle patch/epidermal growth factor (EGF). ${ }^{*} p<0.05$, statistically significant compared with microneedle patches.

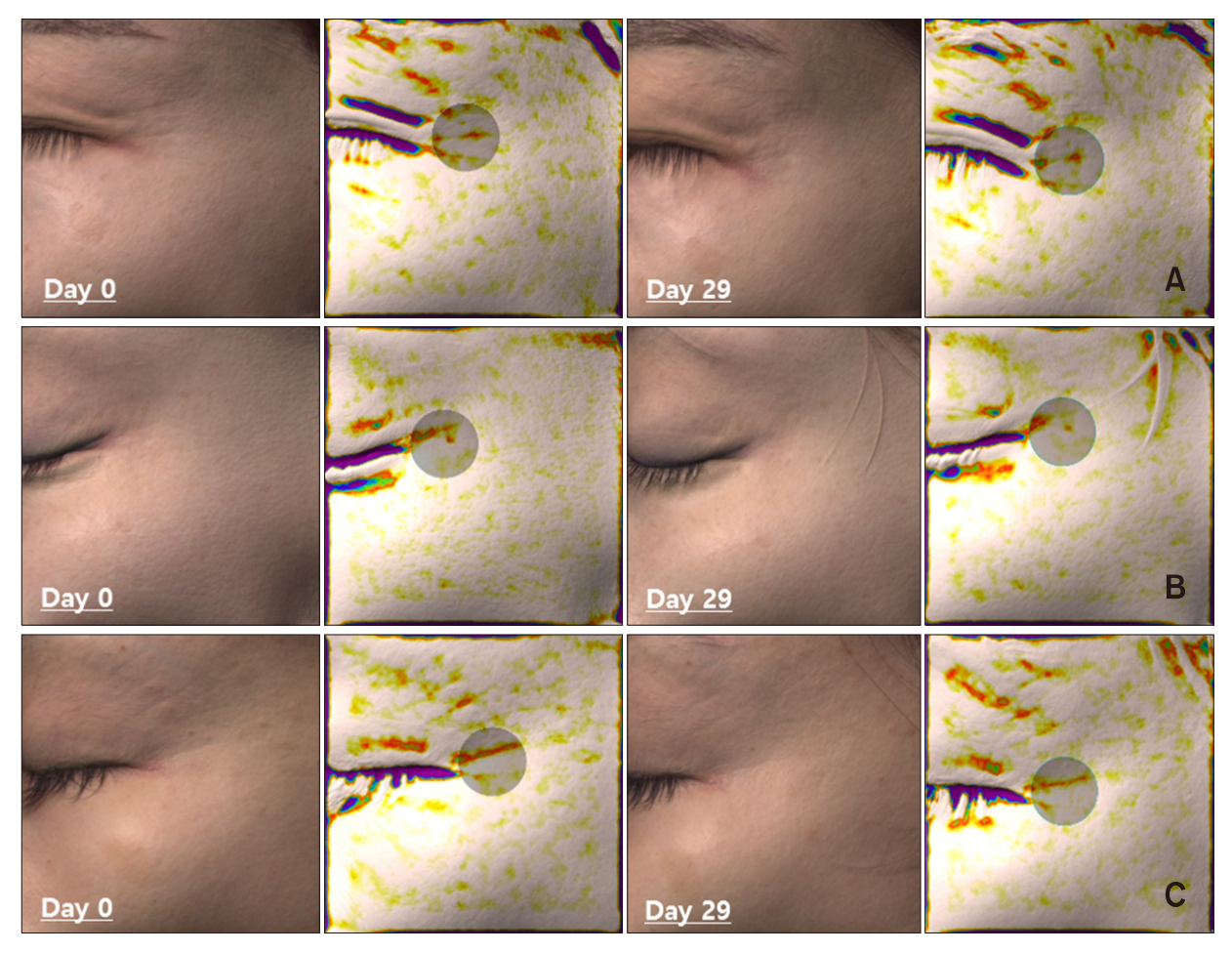

Fig. 7. Photograph and Antera $3 \mathrm{D}^{\mathbb{R}}$ image of a wrinkle at day 0 and day 29 after treatment of microneedle patch (A), microneedle patch/ acetyl hexapeptide-8 (B), and microneedle patch/epidermal growth factor (C).
$60.6 \pm 3.5$ to $68.4 \pm 2.8$ at day 29 after the application of microneedle patch/EGF $(p<0.01)$. Skin hydration increased by $12.3 \%$ from $60.4 \pm 3.4$ to $67.8 \pm 3.1$ at day 29 after the application of microneedle patch alone, too. No significant difference was found in the degree of improvement between the products.

\section{Adverse effects}

No adverse effects were observed during follow-up.

\section{DISCUSSION}

In this study, the anti-wrinkle and skin moisturizing effects of CLHA-based microneedle patch containing AHP-8 and 
EGF were assessed. For assessment of appearances of wrinkle and moisture improvement, direct measurement using the Antera 3D ${ }^{\circledR}$, Corneometer ${ }^{\circledR}$ and photodamage score assessment were used to obtain objective results. The results of Antera 3D ${ }^{\circledR}$ showed statistically significant improvements starting from day 1 to 29 after the application of both microneedle patch/AHP-8 and microneedle patch/EGF $(p<0.01)$. The photodamage score assessment also confirmed significant improvements after the application of both products. The reason why improvements were observed earlier in the direct measurement using a device may be because the Antera $3 D^{\mathbb{R}}$ can assess the skin in more detail. Antera $3 D^{\circledR}$ is a skin imaging analysis instrument for measuring wrinkle, melanin texture. Linming et al. ${ }^{19}$ reported that the Antera $3 \mathrm{D}^{\mathbb{R}}$ has higher sensitivity than VISIA $^{\circledR}$ (Canfield Imaging Systems, Fairfield, NJ, USA), which is a widely used skin imaging analysis instrument. They described that the Antera $3 D^{\circledR}$ is an appropriate instrument for skin aging assessment. According to the indentation index which is used to measure the degree of wrinkles from Antera $3 D^{\mathbb{R}}$, fine to moderate wrinkles around the eyes improved by $12.5 \%(p<0.001), 13.6 \%$ ( $p$ $<0.001)$, and $14.6 \%(p<0.001)$ at day 1,3 , and 5 , respectively, after the application of microneedle patch/ AHP-8. Therefore, the anti-wrinkle effects of microneedle patch/AHP-8 continued after the first application. On the contrary, fine to moderated wrinkles around the eyes improved by $9.4 \%(p<0.01), 8.7 \%(p<0.01)$, and $5.9 \%(p<0.1)$ at day 1,3 , and 5, respectively, after the application of microneedle patch alone. Therefore, the anti-wrinkle effects of the microneedle patch alone diminished over time after the first application.

Deep wrinkles around the eyes improved by $12.3 \%(p<$ $0.001), 15.2 \%(p<0.001)$, and $13.6 \%(p<0.001)$ at day 1 , 3 , and 5, respectively, after the application of microneedle patch/AHP-8. These improved by $12.6 \%(p<0.01), 11.3 \%$ $(p<0.01)$, and $12.9 \%(p<0.001)$ at day 1,3 , and 5 , respectively, after the application of microneedle patch/EGF. Therefore, the anti-wrinkle effects of microneedle patch/ AHP-8 and microneedle patch/EGF continued after the first application. By contrast, the anti-wrinkle effects of microneedle patch alone decreased over time after the first application $(7.8 \%, 7.3 \%[p<0.05]$, and $3.5 \%$ at day 1,3 , and 5 , respectively, after the application). Improvements were statistically significant at day 5 after the application of microneedle patch/AHP-8 and microneedle patch/EGF compared with microneedle patch alone $(p<0.05)$. The photodamage score assessment performed by three dermatologists showed significant improvements starting at day 8 after the application of microneedle patch/AHP-8 or microneedle patch/EGF $(p<0.05)$. In contrast, significant improvements were observed at day 29 after the application of microneedle patch alone $(p<0.05)$. In comparison between groups, on the fifth day, statistically significant effect on fine to moderate wrinkles was confirmed in microneedle patch containing AHP compared to microneedle alone patch. Moreover, statistically significant effect on deep wrinkles was confirmed in microneedle patch containing AHP or EGF compared to microneedle alone patch, on the fifth day. Therefore, these effects are considered to be resulting from both the use of microneedle patch and the additional ingredients including the CLHA. But no significant difference was found in the degree of improvement between the products in photodamage score. These differences may be attributed to the addition of functional materials, AHP-8 and EGF. AHP-8 (Argireline ${ }^{\circledR}$ peptide/original manufacturer, Lipotec $\mathrm{SAU}$ ) is a synthetic peptide patterned from the $\mathrm{N}$-terminal end of synaptosomal-associated protein-25 (SNAP-25) and used to reduce the appearances of wrinkles. AHP-8 can emulate the action of botulinum neurotoxins (BoNTs) to prevent the formation of the ternary soluble $\mathrm{N}$-ethylmaleimide sensitive factor attachment protein receptor (SNARE) complex and interfere with catecholamine release. In accordance with the action of AHP-8, the contraction of the facial intrinsic muscles is inhibited. Even though AHP-8 has lesser longer-lasting anti-wrinkle effects than BoNTs, it has significant potency of inhibition of neurotransmitter release and no neurotoxicity ${ }^{20-22}$. EGF is a polypeptide composed of 53 amino acids that promotes epithelial cell proliferation and division. Moreover, it can significantly enhance the moisturization and wound healing effects. While the mechanism by which wrinkles are improved is unclear, there have been many reports of EGF, EGF receptors, and their associated series of responses. As age increases, EGF receptor decline is suggested as one of the mechanisms of aging. The synthesis of fibronectin and collagen is reported to increase when EGF is administered to fibroblasts. Clinical tests using EGF serum have shown statistically significant improvements in terms of appearance of fine lines and skin texture ${ }^{23,24}$.

In recent years, microneedle patch has been produced via various methods and the effects were assessed. Choi et al. ${ }^{7}$ assessed wrinkle and skin elasticity improvements after the application of microneedle patch containing HA twice per week for 8 weeks and observed statistically significant improvements starting at week 2 . Kim et al. ${ }^{8}$ assessed skin roughness following the application of microneedle patch containing retinoic acid or ascorbic acid twice per day for 12 weeks and reported significant improvements at week 12. When they applied microneedle patch containing HA and EGF once every 2 days for 8 weeks, they observed 
significant improvements in participants at week 8 in wrinkle severity rating scale ${ }^{25}$. Notably, our study observed the effects of microneedle patch after a short period of use although applied less frequently compared with previous studies. This may be because we used microneedle patch containing not only HA but also CLHA. CLHA forms an ether bond with additives that induce cross-linking reactions with the hydroxyl groups of HA and form a crosslinking structure. This cross-link structure is more complicated and compact than the linear structure that HA usually has as it is formed upon the binding of many HA molecules. This structural characteristic reduces the space in which hyaluronidase and free radicals can bind to HA molecules, thereby delaying the rapid degradation of $\mathrm{HA}$, an inherent drawback of HA. Depending on the degree of CLHA whose half-life is increased from half a day to 2 days, HA stays longer, and the effects of the raw material last for a longer time ${ }^{26-29}$. In addition, CLHA forms a hydrogel and swells upon absorbing water; thus, it has better biomechanical properties than does $\mathrm{HA}^{30}$. In recent study, the swelling ratio of CLHA until 24 hours was about 3 to 5 times larger compared to $\mathrm{HA}$, and the degradation ratio of HA or CLHA by hyaluronidase until 100 hours was less than half. However, it should be further considered that the effect of the microneedle patch within few days can be a temporary swelling effect caused by physical stimulation of needling ${ }^{31}$. When supplementary materials, such as AHP-8 and EGF, are mixed with CLHA and $\mathrm{HA}$, their active compounds slowly decompose contrary to when they are mixed with non-CLHA. It may be via this mechanism that the effects of the product can be maintained for a relatively long period of time.

The limitation of this study is that the sample size was small. This study assessed the improvements in skin appearances of wrinkles and moisture after a short-term application of microneedle patch. The results suggest that the application of repeated CLHA-based microneedle patch containing AHP-8 and EGF will have the effect of relatively continuous wrinkle improvement. Further study with larger population may explore longer term follow up to test for the durability. In conclusion, application of CLHAbased microneedle patch containing AHP-8 and EGF showed significant improvements in wrinkle and skin moisturization with a good safety profile.

\section{ACKNOWLEDGMENT}

This research was supported by a grant of the Korea Health Technology R\&D Project through the Korea Health Industry Development Institute (KHIDI), funded by the Ministry of Health \& Welfare, Republic of Korea (grant number: HI16C1559).

\section{CONFLICTS OF INTEREST}

The authors have nothing to disclose.

\section{ORCID}

Ji Hae An, https://orcid.org/0000-0003-0497-9538

Hee Jung Lee, https://orcid.org/0000-0001-9140-9677

Moon Soo Yoon, https://orcid.org/0000-0002-7470-6802

Dong Hyun Kim, https://orcid.org/0000-0003-3394-2400

\section{REFERENCES}

1. Papakonstantinou E, Roth M, Karakiulakis G. Hyaluronic acid: a key molecule in skin aging. Dermatoendocrinol 2012;4:253-258.

2. Prestwich GD, Marecak DM, Marecek JF, Vercruysse KP, Ziebell MR. Controlled chemical modification of hyaluronic acid: synthesis, applications, and biodegradation of hydrazide derivatives. J Control Release 1998;53:93-103.

3. Bulpitt P, Aeschlimann D. New strategy for chemical modification of hyaluronic acid: preparation of functionalized derivatives and their use in the formation of novel biocompatible hydrogels. J Biomed Mater Res 1999;47:152-169.

4. Al-Sibani M, Al-Harrasi A, Neubert RHH. Evaluation of invitro degradation rate of hyaluronic acid-based hydrogel cross-linked with 1, 4-butanediol diglycidyl ether (BDDE) using RP-HPLC and UV-Vis spectroscopy. J Drug Deliv Sci Technol 2015;29:24-30.

5. Kenne L, Gohil S, Nilsson EM, Karlsson A, Ericsson D, Helander Kenne A, et al. Modification and cross-linking parameters in hyaluronic acid hydrogels--definitions and analytical methods. Carbohydr Polym 2013;91:410-418.

6. Liu S, Jin MN, Quan YS, Kamiyama F, Kusamori K, Katsumi $\mathrm{H}$, et al. Transdermal delivery of relatively high molecular weight drugs using novel self-dissolving microneedle arrays fabricated from hyaluronic acid and their characteristics and safety after application to the skin. Eur J Pharm Biopharm 2014;86:267-276.

7. Choi SY, Kwon HJ, Ahn GR, Ko EJ, Yoo KH, Kim BJ, et al. Hyaluronic acid microneedle patch for the improvement of crow's feet wrinkles. Dermatol Ther 2017;30:e12546.

8. Kim $M$, Yang $H$, Kim $H$, Jung $H$, Jung $H$. Novel cosmetic patches for wrinkle improvement: retinyl retinoate- and ascorbic acid-loaded dissolving microneedles. Int J Cosmet Sci 2014;36:207-212.

9. Lee $C$, Yang $H, \operatorname{Kim} S$, Kim $M$, Kang $H$, Kim $N$, et al. Evaluation of the anti-wrinkle effect of an ascorbic acidloaded dissolving microneedle patch via a double-blind, placebo-controlled clinical study. Int J Cosmet Sci 2016;38: 375-381.

10. Hong JY, Ko EJ, Choi SY, Li K, Kim AR, Park JO, et al. Efficacy and safety of a novel, soluble microneedle patch for the improvement of facial wrinkle. J Cosmet Dermatol 
2018;17:235-241.

11. Chung JH, Lee SH, Youn CS, Park BJ, Kim KH, Park KC, et al. Cutaneous photodamage in Koreans: influence of sex, sun exposure, smoking, and skin color. Arch Dermatol 2001;137:1043-1051.

12. Larnier C, Ortonne JP, Venot A, Faivre B, Béani JC, Thomas $\mathrm{P}$, et al. Evaluation of cutaneous photodamage using a photographic scale. Br J Dermatol 1994;130:167-173.

13. Messaraa C, Metois A, Walsh M, Hurley S, Doyle L, Mansfield $A$, et al. Wrinkle and roughness measurement by the Antera 3D and its application for evaluation of cosmetic products. Skin Res Technol 2018;24:359-366.

14. Hamilton JD. Fabrication and analysis of injection molded plastic microneedle arrays [thesis]. Atlanta: Georgia Institute of Technology; 2010.

15. Weindl G, Schaller $M$, Schäfer-Korting $M$, Korting $H C$. Hyaluronic acid in the treatment and prevention of skin diseases: molecular biological, pharmaceutical and clinical aspects. Skin Pharmacol Physiol 2004;17:207-213.

16. Tammi MI, Day AJ, Turley EA. Hyaluronan and homeostasis: a balancing act. J Biol Chem 2002;277:4581-4584.

17. Wang F, Garza LA, Kang S, Varani J, Orringer JS, Fisher GJ, et al. In vivo stimulation of de novo collagen production caused by cross-linked hyaluronic acid dermal filler injections in photodamaged human skin. Arch Dermatol 2007; 143:155-163.

18. Shoshani D, Markovitz E, Monstrey SJ, Narins DJ. The modified Fitzpatrick Wrinkle Scale: a clinical validated measurement tool for nasolabial wrinkle severity assessment. Dermatol Surg 2008;34 Suppl 1:S85-S91; discussion S91.

19. Linming F, Wei H, Anqi L, Yuanyu C, Heng X, Sushmita P, et al. Comparison of two skin imaging analysis instruments: the VISIA ${ }^{\circledR}$ from Canfield vs the ANTERA 3D ${ }^{\circledR}$ CS from Miravex. Skin Res Technol 2018;24:3-8.

20. Blanes-Mira C, Clemente J, Jodas G, Gil A, Fernández-Ballester G, Ponsati B, et al. A synthetic hexapeptide (Argireline) with antiwrinkle activity. Int J Cosmet Sci 2002;24:303-310.

21. Wang $Y$, Wang $M$, Xiao $S$, Pan $P$, Li $P$, Huo J. The antiwrinkle efficacy of argireline, a synthetic hexapeptide, in
Chinese subjects: a randomized, placebo-controlled study. Am J Clin Dermatol 2013;14:147-153.

22. Gutierrez LM, Viniegra S, Rueda J, Ferrer-Montiel AV, Canaves JM, Montal M. A peptide that mimics the C-terminal sequence of SNAP-25 inhibits secretory vesicle docking in chromaffin cells. J Biol Chem 1997;272:2634-2639.

23. Schouest JM, Luu TK, Moy RL. Improved texture and appearance of human facial skin after daily topical application of barley produced, synthetic, human-like epidermal growth factor (EGF) serum. J Drugs Dermatol 2012;11:613-620.

24. An JJ, Eum WS, Kwon HS, Koh JS, Lee SY, Baek JH, et al. Protective effects of skin permeable epidermal and fibroblast growth factor against ultraviolet-induced skin damage and human skin wrinkles. J Cosmet Dermatol 2013;12:287295.

25. Park J, Seo J, Shin JU, Jeong DH, Kim JD, Lee KH. Efficacy of biodegradable microneedle patches on periorbital wrinkles. Korean J Dermatol 2014;52:597-607.

26. Park S, Park KY, Yeo IK, Cho SY, Ah YC, Koh HJ, et al. Investigation of the degradation-retarding effect caused by the low swelling capacity of a novel hyaluronic Acid filler developed by solid-phase crosslinking technology. Ann Dermatol 2014;26:357-362.

27. Fraser JR, Laurent TC, Laurent UB. Hyaluronan: its nature, distribution, functions and turnover. J Intern Med 1997;242: 27-33.

28. De Boulle K, Glogau R, Kono T, Nathan M, Tezel A, RocaMartinez JX, et al. A review of the metabolism of 1,4butanediol diglycidyl ether-crosslinked hyaluronic acid dermal fillers. Dermatol Surg 2013;39:1758-1766.

29. Edsman K, Nord LI, Ohrlund A, Lärkner H, Kenne AH. Gel properties of hyaluronic acid dermal fillers. Dermatol Surg 2012;38(7 Pt 2):1170-1179.

30. Kablik J, Monheit GD, Yu L, Chang G, Gershkovich J. Comparative physical properties of hyaluronic acid dermal fillers. Dermatol Surg 2009;35 Suppl 1:302-312.

31. Choi JT, Park SJ, Park JH. Microneedles containing crosslinked hyaluronic acid particulates for control of degradation and swelling behaviour after administration into skin. J Drug Target 2018;26:884-894. 